\title{
La caminabilidad en Bogotá: propósitos y condiciones socioespaciales que facilitan y limitan esta experiencia
}

\author{
The Walkability of Bogotá: purposes and socio-spatial conditions that facilitate \\ and limit this experience \\ A caminhabilidade em Bogotá: propósitos e condições socioespaciais que \\ facilitam e limitam essa experiência
}

Pablo Páramo

Universidad Pedagógica Nacional. Bogotá (Colombia)

\section{Andrea Burbano}

Universidad Pedagógica Nacional. Bogotá (Colombia)

Páramo, P., y Burbano, A. (2019). La caminabilidad en Bogotá: propósitos y condiciones socioespaciales que facilitan y limitan esta experiencia. Revista de Arquitectura (Bogotá), 21(2), 12-21. doi: http://dx.doi.org/l0.14718/ RevArq.2019.21.2.2642

\section{(c) (i) \$}

ttp://dx.doi.org/10.14718/RevArq.2019.21.2.2642

\section{Pablo Páramo}

Psicólogo, Universidad Católica de Colombia, Bogotá (Colombia).

Master of Science, University of Surrey, Reino Unido.

Ph.D. en Psicología, Centro de Graduados de la Universidad de la Ciudad de Nueva York (EE.UU.)

https://scholar.google.es/citations?user=rGq8GlwAAAAJ\&hl=es

(D) https://orcid.org/0000-0002-455I-3040

pdeparamo@gmail.com

Andrea Burbano

Arquitecta, Universidad de La Salle, Bogotá (Colombia)

Magíster en Gestión Urbana, Universidad Piloto de Colombia, Bogotá (Colombia)

Ph.D. en Estudios Territoriales, Universidad de Caldas, Manizales (Colombia).

https://scholar.google.es/citations?user=DsMthqMAAAA|\&hl=es

(D) https://orcid.org/0000-0003-2037-5603

arqburbano@gmail.com

\section{Resumen}

Diversos trabajos se han aproximado al caminar desde la literatura, la fenomenología, la sociología y la arquitectura; sin embargo, son pocos los que en nuestro medio se han acercado a recoger las valoraciones que hacen los habitantes de una ciudad a partir de su experiencia como peatones. Se presentan los resultados de un estudio descriptivo de tipo correlacional que explora la forma en que los habitantes de Bogotá valoran qué tan caminable es la ciudad. Participaron un total de 305 personas de diversos grupos de edad, género y habitantes de distintas localidades quienes evaluaron, mediante un cuestionario ad hoc constituido por 50 reactivos, las diferentes condiciones tanto sociales como físicas del espacio público relevantes para conseguir que la ciudad sea caminable. Un análisis de escalamiento multidimensional, el Smallest Space Analysis (SSA), permitió establecer algunos factores clave al determinar los propósitos, el lugar por donde se camina y las condiciones socioespaciales asociadas a la experiencia de caminar. Se discuten los resultados a partir de las implicaciones que tiene caminar por los espacios públicos para la calidad de vida urbana, la formulación de políticas, la planeación y gestión urbana.

Palabras clave: calidad de vida urbana; ciudad caminable; espacio público; modelo de ciudad compacta; movilidad urbana; peatones; percepción urbana; urbanismo.

\section{Abstract}

Studies have approached walking from literature, phenomenology, health research, sociology, gender studies, urban management and architecture. However, few in our environment have collected and systematized the assessments of a city's inhabitants based on their experiences as pedestrians. Thus, the present research aimed to shed some light on this topic. The article presents the results of a descriptive, correlational study that explores the way in which Bogota residents assess how walkable the city is. A total of 305 people from diverse age groups, genders, and different city districts participated in the study by responding to a 50 item ad hoc questionnaire to evaluate different social and physical conditions of public space, relevant to making the city walkable. A multidimensional scaling analysis, the Smallest Space Analysis (SSA), allowed researchers to establish some key factors when determining the purposes, the places people walk, and the socio-spatial conditions associated with the walking experience. The results are discussed based on the implications of walking public spaces on the quality of urban life, the formulation of public policies, planning, and urban management.

Keywords: Quality of urban life; walkable city; public space; compact city model; urban mobility; pedestrians; urban perception; urban planning.

\section{Resumo}

Diversos trabalhos têm se aproximado do caminhar a partir da literatura, da fenomenologia, da sociologia e da arquitetura; contudo, são poucos os que, em nosso meio, têm se aproximado das avaliações que os habitantes de uma cidade fazem a partir de sua experiência como pedestres. Neste texto, são apresentados os resultados de um estudo descritivo de tipo correlacional que explora a forma em que os habitantes de Bogotá, Colômbia, avaliam o quão caminhável é a cidade. Participaram 305 pessoas de diversas idades, gênero e habitantes de diferentes setores da cidade, os quais avaliaram, mediante questionário ad hoc constituído por 50 reativos, as diferentes condições tanto sociais quanto físicas do espaço público relevantes para tornar a cidade caminhável. Uma análise de escalonamento multidimensional, a Smallest Space Analysis (SSA), permitiu estabelecer alguns fatores-chave ao determinar os propósitos, o lugar por onde se caminha e as condições socioespaciais associadas à experiência de caminhar. São discutidos os resultados com base nas implicações que o caminhar pelos espaços públicos tem para a qualidade de vida urbana, para a formulação de políticas, para o planejamento urbano e para a gestão urbana.

Palavras-chave: qualidade de vida urbana; cidade caminhável; espaço público; modelo de cidade compacta; mobilidade urbana; pedestres; percepção urbana; urbanismo. 


\section{Introducción}

El presente artículo es resultado del proyecto institucional: "El caminar como experiencia de aprendizaje de la ciudad", financiado por el Centro de Investigaciones de la Universidad Pedagógica Nacional durante el año 2018', el cual tuvo como objetivo: identificar la estructura conceptual mediante la cual los participantes valoran las condiciones físicas y sociales que posibilitan la experiencia de aprehender la ciudad caminando; los propósitos que persiguen las personas al caminar; recoger la experiencia que se adquiere en los lugares por los que se transita y, a partir de esta exploración, formular recomendaciones de gestión para la educación urbana y lineamientos normativos que contribuyan a que la ciudad sea caminable dentro del contexto de ciudad educadora.

El artículo parte de la revisión de algunos trabajos científicos que indagan sobre el caminar desde la perspectiva de los estudios en salud, la seguridad del peatón, el género y la gestión urbana. Posteriormente, justifica la necesidad de adicionar a los indicadores que se han venido construyendo, la exploración de las valoraciones subjetivas que hacen las personas acerca de qué tan caminable es la ciudad. En consecuencia, con este planteamiento se analizan los resultados producto de la aplicación de un cuestionario mediante el cual se recogen las valoraciones que hacen habitantes de Bogotá sobre la caminabilidad de la ciudad y se analizan sus respuestas mediante técnicas estadísticas de escalamiento multidimensional.

\section{El caminar urbano}

Caminar se suele presentar como una actividad motora que contribuye a mantener el estado físico y mejorar las condiciones de salud de las personas. Además de aportar a la movilidad sostenible, caminar en la ciudad propicia los encuentros sociales, recreativos y de consumo con lo que se adquiere una experiencia cultural de apropiación del entorno urbano.

En este artículo se analiza el caminar a partir de la identificación de los propósitos que tienen las personas cuando se desplazan a pie por

Se agradece la colaboración de los estudiantes de la Universidad Pedagógica Nacional, Colombia: Andrés Felipe Colorado, Camilo Moreno y Alexandra Prado. distintos escenarios y sus percepciones sobre las condiciones físicas y sociales que facilitan o dificultan que una ciudad, en este caso Bogotá, sea caminable.

Las investigaciones sobre el caminar se centran en su mayor parte en destacar la importancia para la salud. Las personas que caminan son menos propensas a la obesidad (Bassett, Pucher, Buehler, Thompson y Crouter 2008; Haines et al., 2009) y caminar es ampliamente recomendado como parte de un proceso de rehabilitación cuando se han tenido problemas de salud de distinto tipo (Forjuoh et al., 2017; Keller y Cantue, 2008). Respecto a la recreación y el ejercicio los estudios reconocen la importancia de la recreación al aire libre en entornos urbanos, donde la caminata por el vecindario debe considerarse como una actividad lúdica dentro del marco de la recreación urbana (Lee y Ingold, 2006; Le Breton, 2007; Keller y Cantue, 2008). Sobre la seguridad, las estadísticas muestran que los accidentes de peatones son una de las principales causas de morbilidad y mortalidad en Colombia. Para 2017, el informe de Medicina Legal reportó 7936 casos de accidentes de peatones y 1790 accidentes fatales (Instituto de Medicina Legal y Ciencias Forenses, 2017, p. 389).

Los estudios del diseño espacial y de género merecen especial atención para los propósitos de este artículo. En lo que tiene que ver con las características espaciales y su incidencia sobre el comportamiento, las investigaciones en las que se indaga por la dimensión psicoespacial destacan el efecto en la desaparición de los espacios de encuentro social, que se situaban en las calles y plazas, afectando las dinámicas sociales de encuentro y recreación (Páramo y Cuervo, 2009; 2013). Como lo señaló Jacobs (1961), las aceras y las esquinas fueron lugares importantes para las transacciones sociales en la ciudad, para los encuentros informales de los adultos, el juego de los niños y el encuentro entre adolescentes. Actividades estas que se han reemplazado por el "window shopping" de los centros comerciales o los encuentros en sociolugares, ambos escenarios mediados por el consumo (Páramo, 2011).

En una dirección similar, al explorar las barreras físicas y sociales que se encuentran al caminar, Hollenstein et al. (2016) destacan la presencia de aceras, la existencia de lugares de cruce y de límites de velocidad para los vehículos como condiciones para tomar decisiones sobre ir a un

This article is available in English on the website of Revista de Arquitectura (Bogotá) http://dx.doi.org/10.14718/RevArq.2019.21.2.2642

The Walkability of Bogotá: purposes and socio-spatial conditions that facilitate and limit this experienc. 
lugar caminando. Y D'Álessandro, Appolloni y Capasso (2016) agregan aspectos más subjetivos a esta decisión como la percepción de seguridad y la urbanidad de los habitantes de la ciudad. Entre los beneficios de caminar o montar en bicicleta, según algunos estudios ambientales, están mayor inclusión social, una mayor calidad del aire y menor reducción de tráfico (Haines et al., 2009).

En la investigación de Kerr et al. (2016) se identifican algunas situaciones que las personas reconocen como necesarias para movilizarse por el vecindario y la ciudad a pie o en bicicleta. El caminar se asocia principalmente con la percepción de la densidad residencial, la conectividad de las calles, el acceso a centros comerciales, espacios recreacionales, paradas de buses, la estética de los lugares por donde se camina, la seguridad y la distancia percibida hacia el destino. Desde una perspectiva técnica, en los estudios de gestión urbana hay la tendencia a proponer indicadores e índices objetivos sobre qué tan caminables son las ciudades (Talavera y Soria, 2015; Tribby, Miller, Brown, Werner y Smith, 2016; D'Alessandro, Appolloni y Capasso, 2016; Gutiérrez, Caballero y Escamilla, 2019).

Respecto de la condición de género-mujer se ha evidenciado que caminar para las mujeres resulta no solo riesgoso sino limitado por el diseño espacial y las barreras simbólicas que condicionan el acceso al espacio público (Burbano, 2016; Lindón, 2006). Al restringirse el tránsito principalmente a pie, se generan efectos adversos en la vida social urbana de las mujeres, entre los que se incluyen el abandono, el retraimiento del espacio público y el establecimiento de acciones para poder participar en la vida social urbana (Falú, 2009). Según Ortiz-Guitart (2007), la mujer busca evitar ser agredida y víctima de atracos, acosos y violencia sexual cuando hace presencia en el espacio público. La restricción en el caminar ha llevado a que la mujer naturalice rutinas de movilidad con el propósito de evitar riesgos y opte por tomar caminos conocidos, modificar rutas y horarios para el desplazamiento (Siqueira, 2015), y en otros casos, se recluya en el hogar o, cuando transita por el espacio público, se valga de elementos tecnológicos de vigilancia o defensa (Burbano, 2014 ; 2015). Con estudios como los mencionados, la geografía del género evidencia que para las mujeres no es seguro circular libremente por los espacios públicos dada la invasión a su privacidad reflejada en contactos físicos no consentidos o actos violentos de forma verbal o física, lo cual es consecuente con las formas como se ha gestionado el espacio público urbano desde una visión masculina.

Bogotá ha hecho importantes esfuerzos para dotar la ciudad de infraestructura en ciclovías y andenes para hacer la ciudad caminable (Figura 1), crear normas y programas para incentivar el caminar y proteger la vida del peatón, como es el caso de la campaña "Mírate, todos somos peatones", y la política de seguridad vial "Visión cero" para contrarrestar la situación del peatón como el actor vial más vulnerable. Recientemente ha sido sede de la última conferencia internacional de Walk21 (2018).
Figura 1. Mapa Índice de caminabilidad por UPZ

Fuente: DADEP (2018, Mapa 6, p. 37).

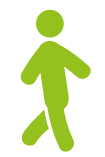

Índice de caminabilidad por UPZ:

En promedio las UPZ tienen un índice de caminabilidad de 0,3626 en un rango de $[0,1]$ con una desviación estándar de 0,08.

El mapa muestra la distribución espacial de los valores calculados del índice de caminabilidad, las UPZ en colores más oscuros muestran los mayores puntajes en el índice, indicando mejores condiciones para la caminabilidad, el top 5 de las UPZ que presentan los mayores valores son en orden: 91-Sangrado Corazón $(0,6)$, 99-Chapinero (0,582), 93-Las Nieves $(0,551)$, 97- Chico Lago $(0,634)$, y Teusaquillo $(0,4982)$.

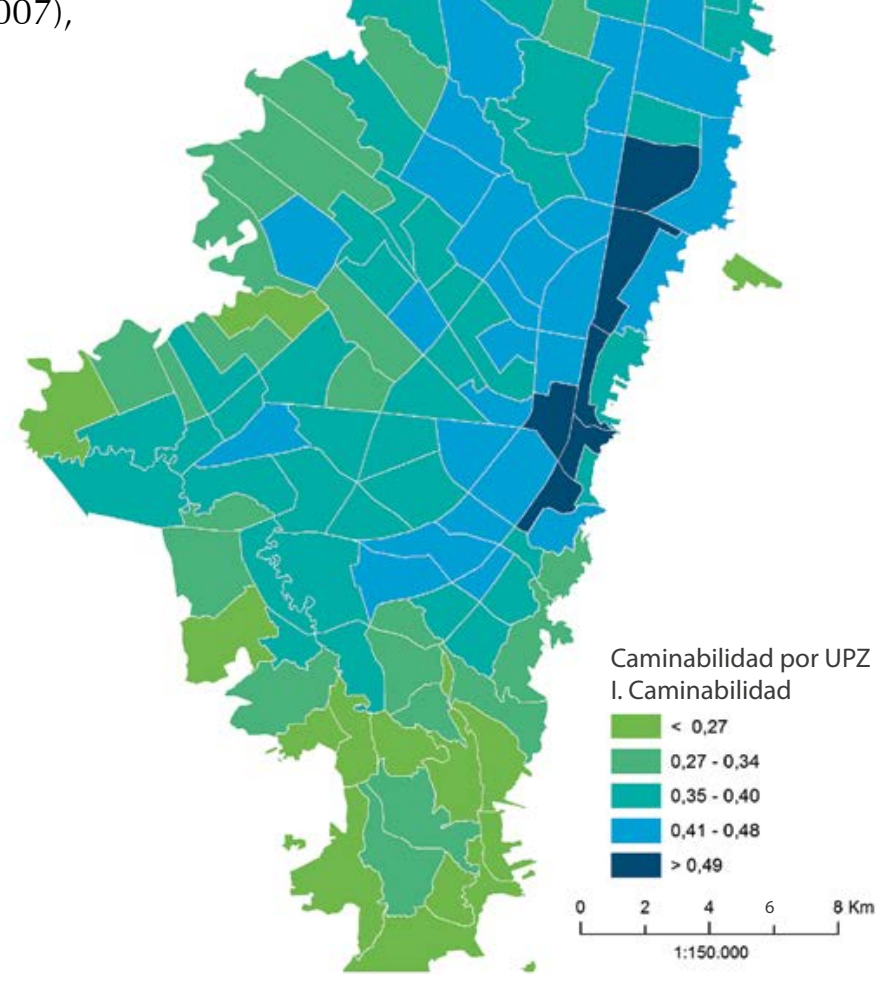


Aun cuando hay una amplia literatura sobre el caminar en los ambientes urbanos, y se han propuesto indicadores objetivos para establecer qué tan caminables son las ciudades, es indispensable explorar las valoraciones subjetivas. Al respecto, es poco lo que se sabe acerca de qué tan caminable es la ciudad para sus habitantes, las motivaciones que tienen para caminar, las dificultades que encuentran al perseguir estos propósitos, y si la experiencia varía dependiendo del lugar por donde de camina.

\section{Metodología}

Diseño. Estudio descriptivo de tipo correlacional, por cuanto se basa en la caracterización de una experiencia recogida mediante afirmaciones que componen un cuestionario, cuyas respuestas son sistematizadas por medio de un progra ma estadístico que permite visualizar el grado de correlación estadística entre los ítems. El diseño sigue las mismas pautas metodológicas que han guiado otros trabajos de los autores (Páramo y Burbano, 2013; Páramo et al., 2018).

Participantes. Participaron voluntariamente 305 personas, hombres y mujeres de diversos grupos de edad y habitantes de distintas localidades de la ciudad. Se utilizó un muestreo de tipo no probabilístico intencional. La muestra fue estratificada de acuerdo con cuatro rangos de edad, desde 18 a 75 años. Un $52 \%$ de los participantes se identificaron como mujeres $(n=166)$, un $44 \%$ como hombres $(n=139)$, ninguno de los participantes se identificó dentro de otro género, a pesar de que el cuestionario daba esta opción. En la tabla 1 se presenta la distribución de la muestra de acuerdo con la cantidad de cuestionarios recogidos y el rango de edad y género informado por los participantes.
V Tabla 1. Distribución de la muestra en Bogotá, según edad y género

Fuente: elaboración propia, 2018.

\begin{tabular}{|c|c|c|c|c|c|c|c|c|c|c|}
\hline \multirow[b]{2}{*}{ Localidad } & \multicolumn{5}{|c|}{ Edad } & \multicolumn{4}{|c|}{ Género } & \multirow[b]{2}{*}{ Total } \\
\hline & $\begin{array}{l}\text { 18-30 } \\
\text { años }\end{array}$ & $\begin{array}{l}\text { 31-45 } \\
\text { años }\end{array}$ & $\begin{array}{l}\text { 46-60 } \\
\text { años }\end{array}$ & $\begin{array}{c}61 \\
\text { años en } \\
\text { adelante }\end{array}$ & $\mathbf{N} / \mathbf{A}$ & Femenino & Masculino & Otro & $\mathbf{N} / \mathbf{A}$ & \\
\hline Ciudad Bolívar & 5 & 12 & 3 & 0 & 0 & 10 & 10 & 0 & 0 & 20 \\
\hline Suba & 24 & 12 & 5 & 4 & 0 & 24 & 21 & 0 & 0 & 45 \\
\hline Engativá & 16 & 12 & 3 & 2 & 0 & 23 & 10 & 0 & 0 & 33 \\
\hline Usaquén & 12 & 4 & 2 & 0 & 0 & 10 & 8 & 0 & 0 & 18 \\
\hline San Cristóbal & 11 & 6 & 4 & 0 & 0 & 11 & 10 & 0 & 0 & 21 \\
\hline Antonio Nariño & 3 & 1 & 0 & 0 & 0 & 0 & 4 & 0 & 0 & 4 \\
\hline Puente Aranda & 8 & 1 & 0 & 0 & 0 & 6 & 3 & 0 & 0 & 9 \\
\hline Chapinero & 3 & 4 & 0 & 1 & 0 & 2 & 6 & 0 & 0 & 8 \\
\hline Teusaquillo & 5 & 3 & 1 & 0 & 0 & 4 & 5 & 0 & 0 & 9 \\
\hline Rafael Uribe & 9 & 4 & 0 & 3 & 0 & 7 & 9 & 0 & 0 & 16 \\
\hline Barrios Unidos & 5 & 3 & 2 & 0 & 0 & 6 & 4 & 0 & 0 & 10 \\
\hline Fontibón & 9 & 2 & 0 & 0 & 0 & 6 & 5 & 0 & 0 & 11 \\
\hline Usme & 3 & 0 & 0 & 0 & 0 & 0 & 3 & 0 & 0 & 3 \\
\hline La Candelaria & 4 & 0 & 0 & 0 & 0 & 3 & 1 & 0 & 0 & 4 \\
\hline Santa Fe & 1 & 0 & 0 & 0 & 0 & 1 & 0 & 0 & 0 & 1 \\
\hline Bosa & 13 & 7 & 0 & 2 & 0 & 12 & 10 & 0 & 0 & 22 \\
\hline $\begin{array}{c}\text { Casos que reportan } \\
\text { información incompleta }\end{array}$ & - & - & - & - & - & 7 & 4 & - & - & 11 \\
\hline Total & 164 & 88 & 25 & 17 & $\mathbf{0}$ & 166 & 139 & $\mathbf{0}$ & $\mathbf{0}$ & 305 \\
\hline Porcentaje & 54 & 29 & 8 & 6 & $\mathbf{0}$ & 52 & 44 & $\mathbf{0}$ & $\mathbf{0}$ & 100 \\
\hline
\end{tabular}


Instrumentos. Se construyó un instrumento ad hoc compuesto por 50 reactivos o ítems que evalúan la caminabilidad de la ciudad a partir de tres dimensiones: el propósito que persigue la persona al caminar, el lugar por donde camina y las condiciones socioespaciales que facilitan o inhiben el caminar, con una escala de siete puntos: desde muy poco caminable (1) a muy caminable (7). El ítem 49 debía ser respondido exclusivamente por mujeres por cuanto indagaba por el acoso al cami- nar. Una escala adicional (ítem 50) preguntaba por la caminabilidad en general de la ciudad por lo que este ítem tuvo un tratamiento diferente. Se incluyó la opción de respuesta "No sabe" para los casos donde la situación por evaluar no aplicaba, según el punto de vista del participante. Los reactivos resultaron de la revisión de la literatura sobre las condiciones que hacen caminable el espacio público urbano y fueron construidos de tal manera que cada uno incluyera en su redac-

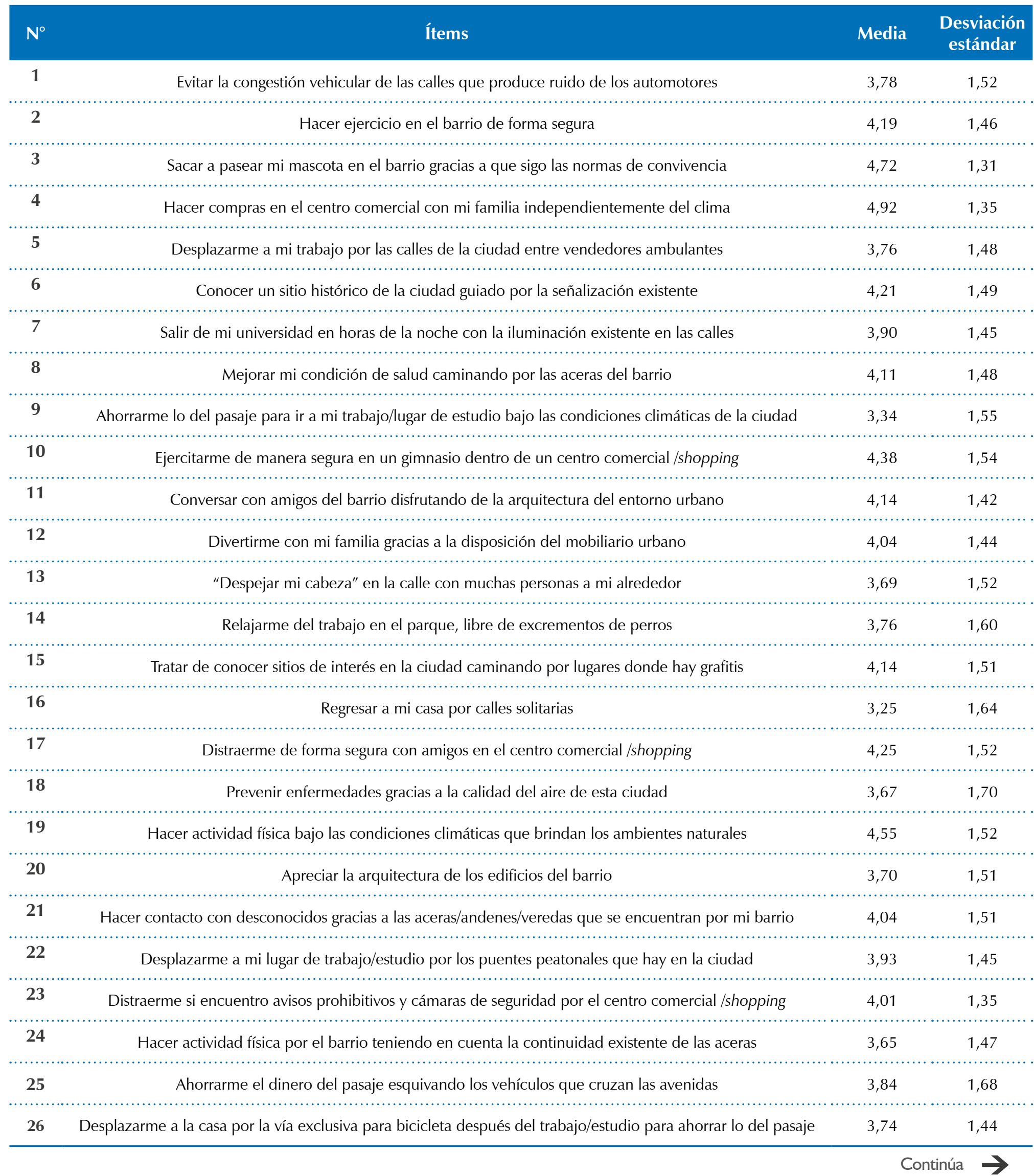


ción un aspecto de cada dimensión por explorar (propósito, escenario y condiciones socioespaciales), siguiendo el modelo de Teoría de Facetas (Borg y Shye, 1995; Hackett, 2014). Al final del instrumento se preguntó sobre las características sociodemográficas del participante, la edad, el género y la localidad de residencia. El cuestionario fue validado por tres jueces independientes y mediante estudio piloto llevado a cabo con 60 participantes. Para el análisis de la consistencia interna del instrumento se utilizó la prueba Alfa de Cronbach cuyo índice estuvo en 0,946.

Procedimiento. El instrumento se aplicó de manera virtual. El consentimiento informado se obtuvo de los participantes al iniciar el diligenciamiento asegurándoles que la información recogida sería completamente anónima y voluntaria. La información fue recolectada durante los meses de abril y mayo de 2018. $\checkmark$ Tabla 2. Promedio y desviación estándar de los ítems ordenados a partir de la media, resultado de la valoración que hicieron los participantes del estudio Fuente: elaboración propia, 2018

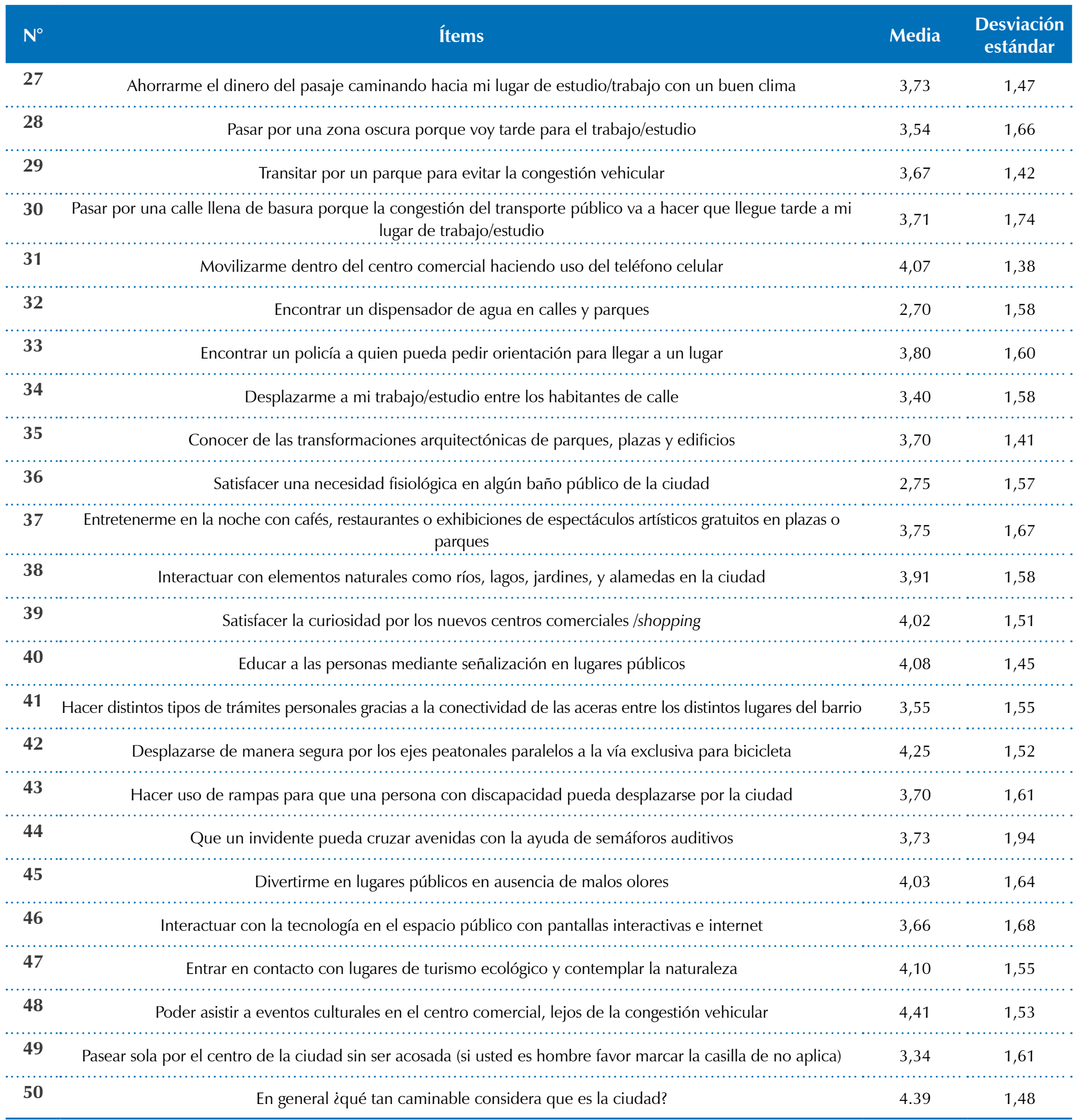




\section{Resultados}

(1) Figura 2. Análisis de distancias mínimas espaciales (SSA) de la dimensión Fuente: elaboración propia, 2018 "Propósitos del caminar"

Con el fin de comparar las valoraciones de los participantes sobre la caminabilidad de la ciudad, se obtuvieron inicialmente los valores de las medias estadísticas y desviaciones estándar para cada ítem (Tabla 2).

\section{Bogota-Propósito}

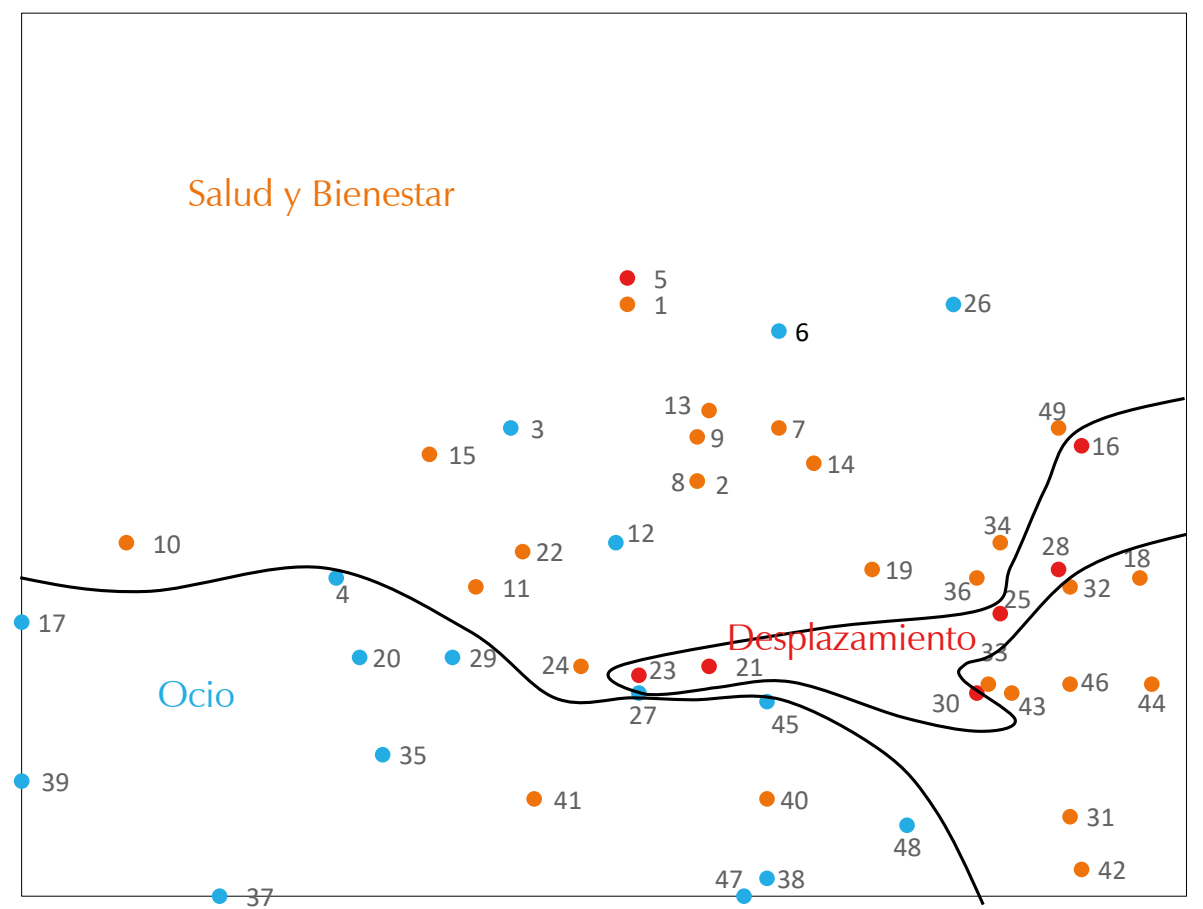

Bogota-Lugar

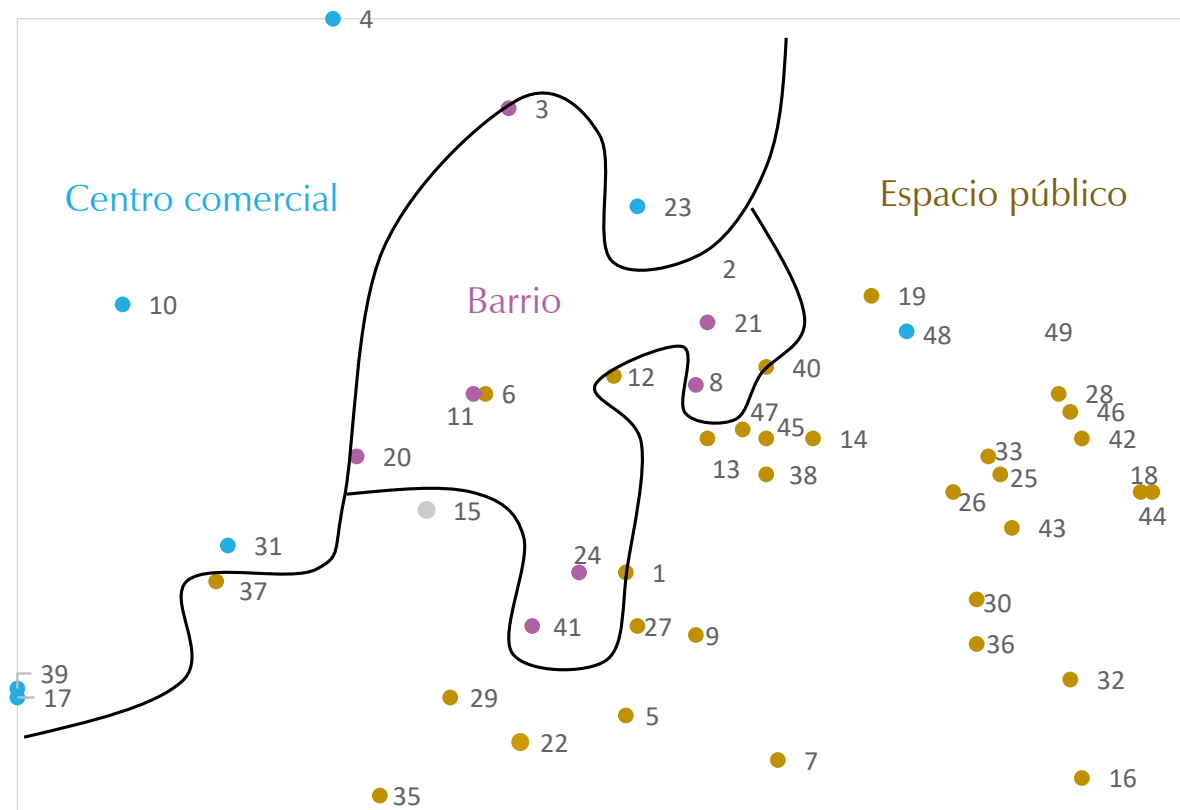

A Figura 3. Análisis de distancias mínimas espaciales (SSA) de la dimensión "Lugar por donde se camina"

Fuente: elaboración propia, 2018.

\section{Análisis de la información mediante el escalamiento multidimensional}

Con el propósito de explorar si los participantes estructuran conceptualmente su valoración de las condiciones asociadas a la caminabilidad de la ciudad a partir de las dimensiones propuestas, las respuestas a los distintos ítems fueron examinadas mediante un análisis de distancias mínimas, Smallest Space Analysis (SSA) (Borg y Groenen, 2005), a través del paquete estadístico HUDAP ${ }^{\circledR}$. El SSA correlaciona los ítems siguiendo las especificaciones del coeficiente de Pearson2, lo que permite visualizar el grado de correlación entre los ítems a partir de su cercanía espacial entre los distintos ítems. El programa arroja tres planos diferentes, uno para cada dimensión a la que hace alusión el ítem. Como se recordará, cada ítem fue diseñado abarcando tres dimensiones (propósitos, lugar donde se camina y condiciones socioespaciales). Las correlaciones entre los ítems resultantes del análisis SSA, que arrojó el programa, permitieron señalar regiones significativas en los respectivos planos o figuras para cada una de las dimensiones exploradas con el cuestionario.

\section{Propósitos}

En la Figura 2 se muestra la distribución espacial de los ítems de acuerdo con el grado de correlación entre ellos a partir de la dimensión "Propósitos del caminar". Se puede observar que estos se agrupan en tres regiones. Una primera, denominada ocio, agrupa ítems que se refieren a esta activad. Así, por ejemplo, se encuentran en esta región los ítems: 4) hacer compras en el centro comercial con mi familia; 17) distraerme de forma segura con amigos en el centro comercial/shopping; 48) poder asistir a eventos culturales en el centro comercial, los cuales tienen las medias más altas. Una segunda región, en la parte superior del gráfico, agrupa principalmente ítems que se refieren a la salud y el bienestar, aunque con algunos que bien podrían hacer parte del ocio. Se destacan los ítems: 8) mejorar mi condición de salud caminando por las aceras del barrio; 10) ejercitarme de manera segura en un gimnasio dentro de un centro comercial; 19) hacer actividad física bajo las condiciones climáticas que brindan los ambientes naturales, ítems que cuentan con las medias más altas de

2 Los resultados de la correlación de Pearson que arroja el programa SSA permiten observar los ítems como puntos geométricos, de tal forma que, entre más similares en términos de la correlación entre ellos, más cerca se encontrarán espacialmente. El SSA calcula inicialmente las correlaciones de todas las combinaciones entre los ítems que están representados y produce una matriz de correlaciones la cual se convierte luego en una matriz de distancias, de tal forma que, a mayor correlación entre dos ítems, menor será la distancia entre ellas y viceversa. 
Bogotá-Socioespacial

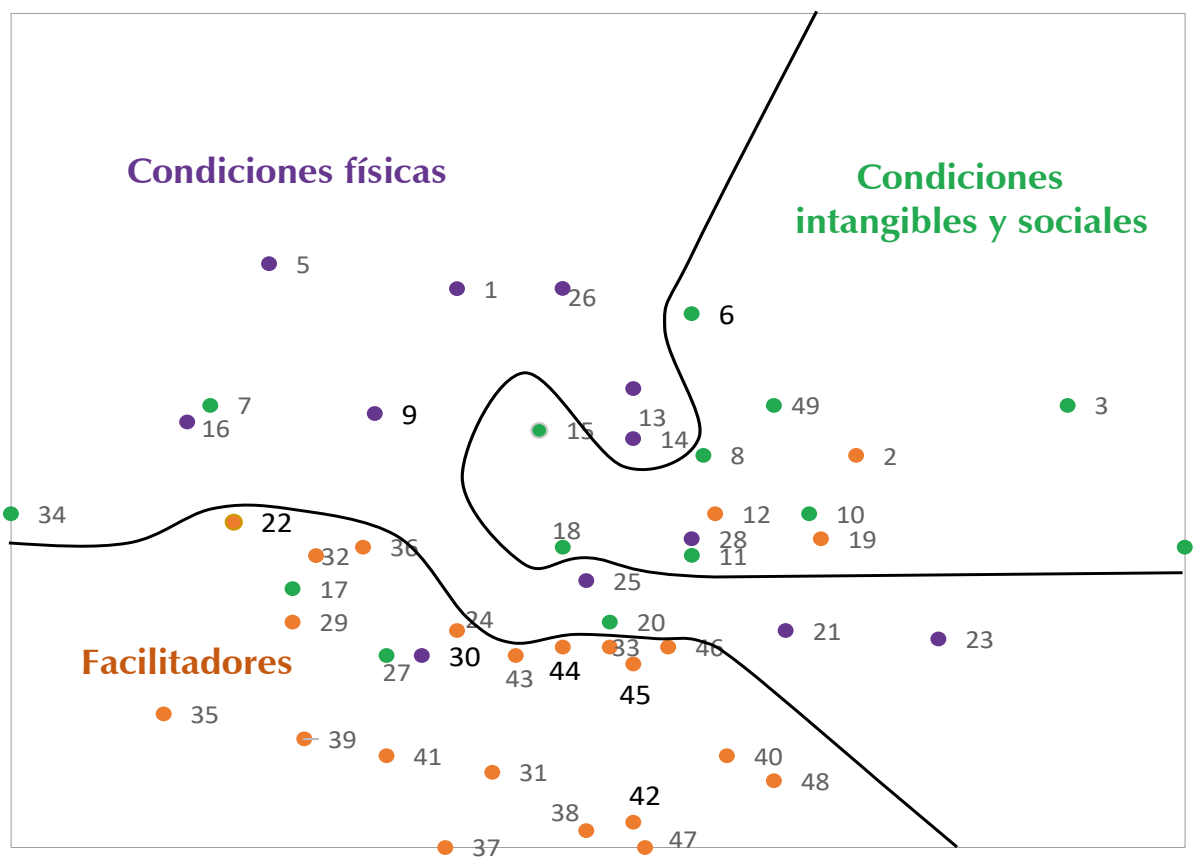

Figura 4. Análisis de distancias mínimas espaciales (SSA) de la dimensión "Condiciones físicas y sociales"

Fuente: elaboración propia, 2018 esta región. Y los ítems: 32) encontrar un dispensador de agua en calles y parques; 36) satisfacer una necesidad fisiológica en algún baño público de la ciudad; y 49) pasear sola por el centro de la ciudad sin ser acosada (Figura 2).

\section{Lugar por donde se camina}

Al identificar los ítems dentro de la dimensión "Lugar por donde se camina", el plano que arrojó el programa SSA se pudo dividir en tres regiones claramente diferenciadas (Figura 3). La primera región, denominada centro comercial, reúne los ítems que hacen referencia a la experiencia de caminar por este tipo de lugares. Sobresalen con medias altas los ítems: 4) hacer compras en el centro comercial con mi familia independientemente del clima; 10) ejercitarme de manera segura en un gimnasio dentro de un centro comercial /shopping; y 17) distraerme de forma segura con amigos en el centro comercial/shopping. En la región central del gráfico se agrupan los ítems que hacen alusión al barrio. Aquí se destaca únicamente el ítem 8) mejorar mi condición de salud caminando por las aceras del barrio, con media alta. Y, finalmente, en la parte derecha e inferior del gráfico se agrupan todos los ítems que hacen alusión al caminar por espacios públicos, sean naturales o construidos. El ítem 19) hacer actividad física bajo las condiciones climáticas que brindan los ambientes naturales, es el que se destaca con un valor alto en la media. Es importante hacer notar que los ítems relacionados con el centro comercial están opuestos espacialmente a los que se refieren a los espacios públicos abiertos, lo que denota correlaciones negativas entre ellos, por lo que puede suponerse que los par- ticipantes consideran opuesta la experiencia entre estos lugares, atribuible muy probablemente a las condiciones de seguridad, encerramiento y diversidad en la percibida. Los ítems con más baja media en la región de espacio público son: 16) regresar a mi casa por calles solitarias; 32) encontrar un dispensador de agua en calles y parques; 36) satisfacer una necesidad fisiológica en algún baño público de la ciudad; y 49) pasear sola por el centro de la ciudad sin ser acosada.

\section{Condiciones socioespaciales que} facilitan o inhiben el caminar

En la figura 4 se pueden observar los ítems diferenciados, ahora, a partir de la dimensión: "Condiciones físicas y sociales", agrupados igualmente en tres regiones. En la primera, ubicada en la parte superior izquierda de la figura, a la que se denomina física, no se encuentran ítems que se destaquen con medias altas. Mientras que el ítem 16) regresar a mi casa por calles solitarias, presenta la media más baja de esta región. En la parte superior derecha se observan los ítems que tienen que ver con las condiciones intangibles y sociales que favorecen el caminar, en donde se destacan por sus medias altas los ítems: 6, 8, 10 y 15 . Y en la parte inferior izquierda se agrupan aquellos ítems que se constituyen en facilitadores del caminar. Se destaca por su media alta el ítem 48.

Se observa una oposición, o correlaciones débiles, entre los ítems que hacen parte de la región facilitadores con la de condiciones sociales, lo que refleja las diferencias entre el papel que juega el ambiente físico frente a los elementos sociales asociados al caminar. Los ítems que presentan una más baja media en la región de facilitadores son el 32 y el 36 . 
Finalmente, respecto a la percepción sobre el acoso que experimentan las mujeres al caminar por los espacios públicos (ítem 49) la media de las 166 participantes para este ítem fue de 3,34, lo que indica que las mujeres se sienten acosadas al desplazarse caminando por Bogotá, situación que las pone en desventaja frente a los hombres y limita su experiencia de apropiación de la ciudad. Y al evaluar en general el grado de caminabilidad de la ciudad (ítem 50), el promedio observado fue de 4,39, lo que indica que para los participantes la ciudad es medianamente caminable.

\section{Discusión y conclusiones}

El estudio identificó tres principales propósitos por los cuales la gente camina: por desplazamiento al lugar de trabajo, de estudio, o para hacer diligencias personales; por ocio o recreación; y por salud y bienestar. Se encontró igualmente la diferenciación por parte de los participantes de la experiencia de caminar entre tres tipos de escenarios: el centro comercial, el barrio y los espacios públicos, naturales y construidos. Y, finalmente, la identificación de condiciones físicas y sociales de caminar: condiciones físicas, condiciones intangibles y sociales, y facilitadores.

Los ítems de más alta valoración son los que afirman que la ciudad es caminable en los centros comerciales y para pasear al perro; las mascotas parecieran ser importantes facilitadores de la salud de las personas. De igual manera, consideran favorables para caminar los elementos naturales con que cuenta la ciudad. Los ítems evaluados menos favorablemente tienen que ver con la seguridad, el acoso que experimentan las mujeres y la falta de elementos del mobiliario urbano como baños públicos y dispensadores de agua.

Se ve así que caminar es más que una experiencia motriz; es social, recreativa, fenomenológica y emocional, por lo que las ciudades no se deben diseñar solo para que sean vistas, sino para ser vividas y habitadas. Lograr que las ciudades sean caminables puede ser un paso importante que conduce a alcanzar este propósito. Por esto, hacer caminable la ciudad se constituye en la mejor opción para integrarse a la ciudad, a la sociedad, mejorar las condiciones de salud, recrearse y adquirir una experiencia significativa por la posibilidad de acceder a ella y apropiársela culturalmente.

Al identificar las razones que motivan a las personas a caminar, y las condiciones espaciales y sociales que lo facilitan o restringen, se hace una contribución importante para mejorar la calidad de vida urbana. Sin lugar a dudas, caminar puede resultar una experiencia enriquecedora en términos formativos, sociales, recreativos, de salud física y mental.

Los hallazgos del estudio son consistentes con las investigaciones revisadas en la introducción del presente artículo al coincidir en que para hacer las ciudades caminables se necesita que sean más seguras, con mayor infraestructura, mayor número de kilómetros caminables y a pequeña escala, y que incluya elementos paisajísticos, naturales y de estética arquitectónica. Pero no basta con la infraestructura física, es importante que se diseñen programas en las escuelas y los organismos de salud y deporte para que las personas caminen más.

Aunque todos somos peatones, no hay políticas claras y suficientes que tengan en cuenta esta condición, lo que afecta de manera indirecta la salud de las personas, y de manera directa cuando se trata de la seguridad. En este sentido, resulta importante contar con índices, indicadores objetivos y subjetivos que permitan hacerle seguimiento a las políticas públicas y a los distintos programas que se adelantan desde la planeación y la gestión urbana.

Tanto Bogotá como el país deben adoptar y crear indicadores objetivos como los propuestos por Gutiérrez, Caballero y Escamilla (2019), que incluyen la calidad ambiental, la densidad poblacional, la proximidad o distancia a sitios de interés, las condiciones físicas de las áreas por donde se camina y la diversidad de usos; y subjetivos, como los aquí señalados, que permitan hacerle seguimiento a las políticas públicas que afectan el caminar.

Resulta fundamental hacer la ciudad más segura mediante el diseño urbano, hacer cumplir el código de policía y educar a la ciudadanía para que las mujeres en particular se sientan confiadas cuando se desplazan caminando por los espacios públicos.

Aunque las mediciones mediante indicadores objetivos sobre el ambiente físico son importantes, las valoraciones que hacen los ciudadanos sobre su experiencia de caminar, a través de indicadores subjetivos, dan información valiosa en la medida en que responden a las necesidades percibidas por la población. Aspectos tales como la percepción de seguridad, la estética, la socialización y las experiencias fenomenológicas de caminar no pueden medirse de forma objetiva pero sí juegan un papel importante en lo que desean los ciudadanos para mejorar su calidad de vida en la ciudad. Los datos son imprescindibles para diagnosticar problemas, diseñar estrategias para solucionarlos y evaluar sus logros. 
Para futuras investigaciones, y debido a la heterogeneidad del territorio, será necesario continuar la línea de investigación de la caminabilidad a partir del barrio, la localidad y el grupo de edad de las personas, lo que requeriría valerse de un muestreo estratificado por barrio, localidad y grupo de edad, en la medida que podrían observarse diferencias importantes que este estudio no exploró dada la modalidad de aplicación del cuestionario. De igual manera, es importante indagar acerca de la experiencia subjetiva de caminar mediante narraciones, en particular y manera más profunda, sobre las valoraciones que hacen las mujeres cuando caminan por la ciudad.

\section{Referencias}

Bassett, D. R. Jr, Pucher, J., Buehler, R., Thompson, D. L. y Crouter, S. E. (2008). Walking, cycling, and obesityrates in Europe, North America, and Australia. Journal of physical activity \& health, 5(6), 795-814. Recuperado de https://www.ncbi.nlm.nih.gov/pub$\mathrm{med} / 19164816$

Borg, I. y Groen, P. J. F. (2005). Modern multidimensional scaling ( 2 a ed.). New York: Springer.

Borg, I. y Shye, S. (1995). Facet Theory: Form and Content. Newbury Park, Ca: Sage.

Burbano, A. (2014). El espacio público urbano situado en la ciudad latinoamericana contemporánea: una aproximación a su estudio desde la perspectiva del género. En E. Licona (coord). Espacio y espacio público contribuciones para su estudio (pp. 151167). Puebla: Benemérita Universidad de Puebla.

Burbano, A. (2015). Mujer y transporte público. En M. C. Aguilar (ed.). Avances de la psicología ambiental ante la promoción de la salud, el bienestar y la calidad de vida (pp. 100107). Granada: Universidad de Granada. Recuperado de https://www.researchgate. net/publication/282610951 AVANCES DE_LA_PSICOLOGIA_AMBIENTTAL_ANTE_LA PROMOCION DE LA SALŪD EL BIENESTAR_Y_LA_CALIDAD_DE_VIDA

Burbano, A. (2016). Modelo territorial para el estudio del espacio público urbano. El caso de una ciudad de Latinoamérica contemporánea (1990-2012) (Tesis Doctoral). Universidad de Caldas, Manizales (Colombia).

D'Alessandro, D., Appolloni, L. y Capasso, L. (2016). How walkableis the city? Application of the walking suitability index of the territory (T-WSI) to the city of Rieti (Lazio Region, Central Italy). Epidemiologia e prevenzione, 40(3-4), 237. https://doi. org/10.19191/EP16.3-4.P237.090

Departamento Administrativo de la Defensoría del Espacio Público (DADEP). (2018). Reporte técnico de indicadores de espacio público 2018. Bogotá: Departamento Administrativo de la Defensoría del Espacio Público. Recuperado de http://observatorio.dadep.gov.co/ sites/default/files/Reporte\%20T\%C3\%A9cnico\%20de\%20Indicadores\%20de\%20Espacio\%20P\%C3\%BAblico\%202018.pdf

Falú, A. (2009). Violencias y discriminaciones en las ciudades. En A. Falú (ed.). Mujeres en la ciudad. De violencias y derechos. Santiago de Chile: Red Mujer y Hábitat de América Latina Ediciones SUR. Recuperado de http:// americalatinagenera.org/newsite/images/ cdr-documents/publicaciones/libro_mujeres_en_la_ciudad.pdf

Forjuoh, S. N., Ory, M. G., Won, J., Towne, S. D., Wang, S. y Lee, C. (2017). Determinants of walkingamong middle-aged and older over- weight and obese adults: Sociodemographic, health, and built environmental factors. Journal of Obesity, 9565430. https://doi. org/10.1155/2017/9565430

Gutiérrez-López, J. A., Caballero-Pérez, Y. B. y Escamilla-Triana, R. A. (2019). Índice de caminabilidad para la ciudad de Bogotá. Revista de Arquitectura (Bogotá), 21(1), 8-20. http://dx. doi.org/10.14718/RevArq.2019.21.1.1884

Hackett, P. M. W. (2014). Facet Theory and the Mapping Sentence: Evolving Philosophy, Use and Application. Basingstoke: Palgrave.

Haines, A., McMichael, A. J., Smith, K. R., Roberts, I., Woodcock, J., Markandya, A. et al. (2009). Public health benefits of strategies to reduce greenhouse-gas emissions: Overview and implications for policy makers. Lancet, 374, 2104-2114. https://doi.org/10.1016/ S0140-6736(09)61759-1

Hollenstein, D. y Bleisch, S. (2016). Walkability for different urban granularities. The International Archives of the Photogrammetry, Remote Sensing and Spatial Information Sciences, XLI-B2. XXIII ISPRS Congress, 12-19 July 2016, Prague. https://doi.org/10.5194/ isprsarchives-XLI-B2-703-2016

Instituto de Medicina Legal y Ciencias Forenses (2018). Datos para la vida. Herramienta para la interpretación, intervención y prevención de lesiones de causa externa en Colombia. Forensis 2017, 19(1), 387-440. Recuperado de http://www.medicinalegal. gov.co/documents/20143/262076/Forensis $+2017+$ Interactivo.pdf

Jacobs, J. (1961). The death and life of great American cities. New York: Vintage Books.

Keller, C. y Cantue, A. (2008). Camina por Salud. Walking in Mexican-American women. Applied Nursing Research, 21(2), 110-113. https://doi.org/10.1016/j.apnr.2006.12.003

Kerr, J., Emond, J. A., Badland, H., Reis, R., Sarmiento, O., Carlson et al. (2016). Perceived neighborhood environmental attributes associated with walking and cycling for transport among adult residents of 17 cities in 12 countries: The IPEN study. Environ Health Perspect, 124(3), 290-8. https://doi. org/10.1289/ehp.1409466

Le Breton, D. (2007). El sabor del mundo. Una antropología de los sentidos. Buenos Aires: Nueva Visión.

Lee, J. e Ingold, T. (2006). Fieldwork on foot: Perceiving, routing, socializing. En P. Collins y S. Coleman (eds.). Locating the Field. Space, Place and Context in Anthropology (pp. 67-86). Berg: Oxford

Lindón, A. (2006). Territorialidad y género, una aproximación desde la subjetividad espacial. En P. Ramírez y M. A. Díaz (coords.). Pensar y habitar la ciudad: afectividad, memoria y significado en el espacio urbano.
Ciudad de México: Anthropos y Universidad Autónoma Metropolitana, Unidad Iztapalapa. Recuperado de http://ru.iis.sociales.unam.mx/jspui/bitstream/IIS/4146/2/ Pensar la ciudad de lugares desde el espacio_pūblico_en_cēentro_historico.pōff

Ortiz-Guitart, A. (2007). Hacia una ciudad no sexista. Algunas reflexiones a partir de la geografía humana feminista para la planeación del espacio urbano. Territorios, (1617).11-28. Recuperado de https://revistas. urosario.edu.co/index.php/territorios/article/view/838

Páramo, P. y Cuervo, M. (2009). La experiencia urbana en el espacio público de Bogotá. Bogotá: Fondo Editorial Universidad Pedagógica Nacional-Ediciones Universidad Santo Tomás.

Páramo, P. (2011). Sociolugares. Bogotá: Universidad Piloto de Colombia.

Páramo, P. y Cuervo, M. (2013). Historia social situada en el espacio público de Bogotá desde su fundación hasta el siglo XIX. Bogotá: Universidad Pedagógica Nacional.

Páramo, P. y Burbano, A. (2013). Valoración de las condiciones que hacen habitable el espacio público en Colombia. Territorios, (28), 187-206. Recuperado de https://revistas.urosario.edu.co/index.php/territorios/ article/view/2557

Páramo, P., Burbano, A., Jiménez-Domínguez, B., Barrios, V., Pasquali, C., Vivas, F. et al. (2018). La habitabilidad del espacio público en las ciudades de América Latina. Avances en psicología latinoamericana, 36(2), 345362. http://dx.doi.org/10.12804/revistas. urosario.edu.co/apl/a.4874

Siqueira, L. de A. (2015). Por onde andam as mulheres: percursos e medos que limitam a experiência de mulheres no centro do Recife (Dissertação Mestrado). Universidade Federal de Pernambuco. Centro de Artes e Comunicação. Desenvolvimento Urbano. Recuperado de https://repositorio.ufpe.br/ handle/123456789/17274

Talavera-García, R. y Soria-Lara, J. A. (2015). Q-PLOS, developing an alternative walking index. A method based on urban design quality. Cities, 4, 7-17. https://doi.org/10.1016/j. cities.2015.03.003

Tribby, C. P., Miller, H. J., Brown, B. B., Werner, C. M. y Smith, K. R. (2016). Assessing built environment walkability using activity-space summary measures. J Transp Land Use, 9, 187207. https://doi.org/10.5198/jtlu.2015.625

Walk21 - Leading the Walking Movement (2018). Walk21- XIX: Bogotá, Colombia. Walkable City, 15-19 de octubre de 2018. Recuperado de https://www.walk21.com/bogota 



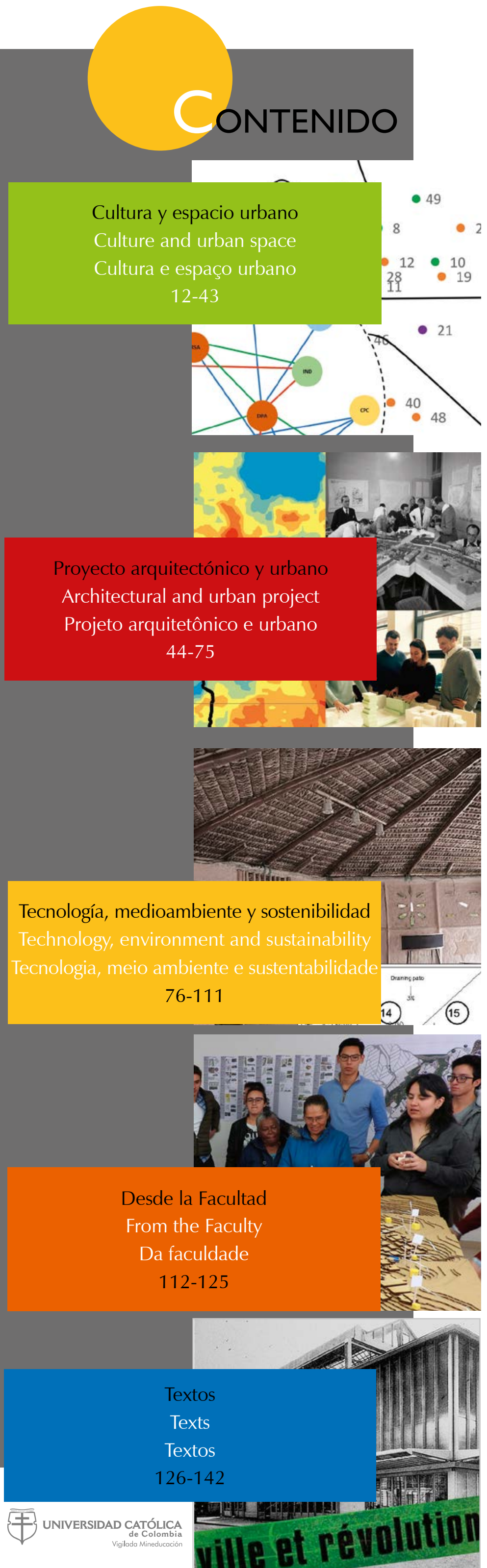

Arquitecturas colectivas y participación como estrategias para la construcción de la ciudad latinoamericana

Myriam Stella Díaz-Osorio

Pág. 3

ES

La caminabilidad en Bogotá: propósitos y condiciones socioespaciales que facilitan y limitan esta experiencia Pablo Páramo

Andrea Burbano

Pág. 12

ES EN

Planificación comunitaria en barrios socialmente

vulnerables. Identificación de los actores sociales en una comunidad

Rafael Alejandro Tavares-Martínez
Jesús Manuel Fitch-Osuna

ES

Pág. 22

Desvanecimiento de la frontera como límite. Imaginario del borde como espacio público físico y virtual

Gabriela Eloísa Muñoz-Torres

Susana Gutiérrez-Luna

Pág. 33

Estudiantes latinoamericanos en el Institut

d'Urbanisme de l'Université de Paris (1923-1941)

$$
\begin{aligned}
& \text { Andrés Ávila-Gómez } \quad \text { Pág. } 44 \\
& \text { ES }
\end{aligned}
$$

Apuntes para el repensamiento de la enseñanza de la Arquitectura. La cuestión epistemológica y la necesidad de una razón ampliada
Juan J. Álvarez-Álvarez
Pág. 57

ES

Equipamientos colectivos: "lugares" de producción de capital social
José Mario Mayorga-Henao
Pág. 68

\section{ES}

Bucle multidisciplinar para la sustentabilidad urbana

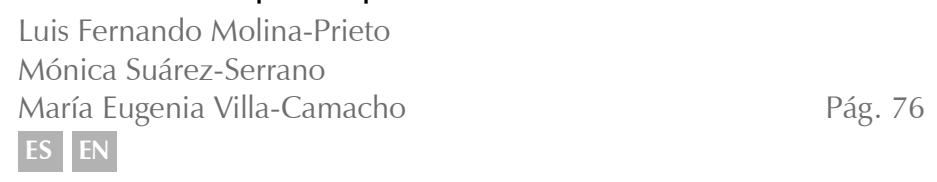

Durabilidad de los materiales naturales de construcción: percepciones de proyectistas, constructores y usuarios en Florianópolis, Brasil

Andrea Salomé Jaramillo-Benavides
Zuleica Maria Patricio-Karnopp
Lisiane Ilha-Librelotto

Pág. 89

\section{ES}

Thermal comfort in buildings for wet processing of coffee

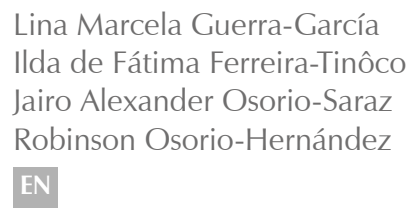

La arquitectura en los barrios: puntos de encuentro entre la academia y el saber popular

Hernando Carvajalino-Bayona

Pág. 112 ES

Arquitectura, modernidad, modernización

$$
\begin{aligned}
& \text { Jean-Louis Cohen } \\
& \text { Traductores } \\
& \text { Andrés Avila-Gómez. } 126 \\
& \text { Diana Carolina Ruiz } \\
& \text { ES }
\end{aligned}
$$


Arquitecturas colectivas y participación como estrategias para la

Arquiteturas coletivas e participação como estratégias para construir a cidad latino-americana

\section{Stella Díaz-Osorio}

La caminabilidad en Bogotá: propósitos y condiciones socioespaciales que facilitan y limitan esta experiencia

The Walkability of Bogotá: purposes and socio-spatial conditions that facilitate and limit this experience

A caminhabilidade em Bogotá: propósitos e condições socioespaciais que facilitam e limitam essa experiência

\section{Andrea Burbano}

Planificación comunitaria en barrios socialmente vulnerables.

Identificación de los actores sociales en una comunidad

Community planning in socially vulnerable neighborhoods.

Identification of social actors in a community

Planejamento comunitário em bairros socialmente vulneráveis.

Identificação dos atores sociais em uma comunidade

Rafael Alejandro Tavares-Martínez

esús Manuel Fitch-Osuna

Desvanecimiento de la frontera como límite. Imaginario del borde como espacio público físico y virtual

$m$

迹

Durabilidad de los materiales naturales de construcción: percepciones de proyectistas, constructores y usuarios en Florianópolis, Brasil

Durability of natural building materials: Perceptions of designers, builders, and users in Florianópolis, Brazil

Durabilidade dos materiais naturais de construção: percepções de projetistas, construtores e usuários em Florianópolis, Brasil

\section{Zuleica Maria Patricio-Karnopp}

Lisiane Ilha-Librelotto

Thermal comfort in buildings for wet processing of coffee

\section{Confort térmico en edificaciones para procesamiento húmedo de café}

Conforto térmico em instalaçōes para processamento úmido de café

\section{Ida de Fátima Ferve-Tartióco}

airo Alexander Osorio-Saraz

Robinson Osorio-Hernandez

La arquitectura en los barrios: puntos de encuentro entre la academia y el saber popular

Architecture in neighborhoods: meeting points between academia and popular knowledge

A arquitetura nos bairros: pontos de encontro entre a academia e o saber popular Hernando Carvajalino-Bayona

\section{Arquitectura, modernidad, modernización}

Architecture, modernity, modernization

Arquitetura, modernidade, modernização
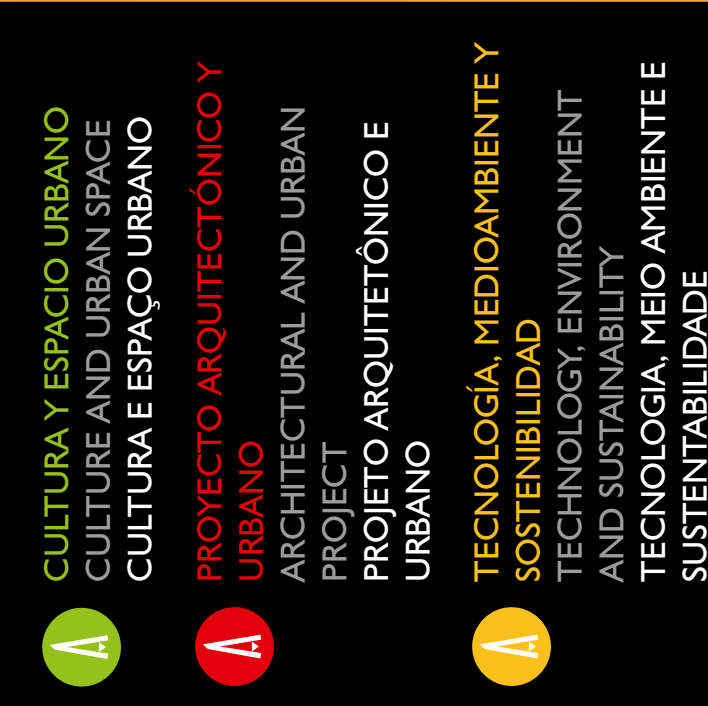

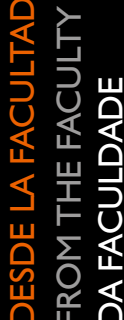

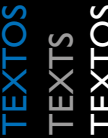
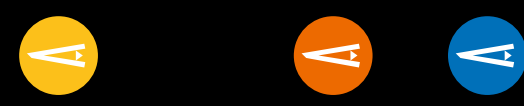

1) Clarivate

publind

Clarivate
Analytics

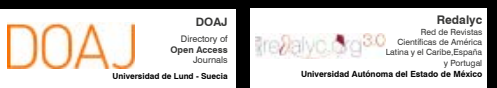

n.

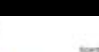

REDIB

EBSCO

cis

latindex unmoxom

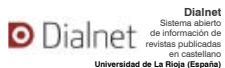

LatinREV

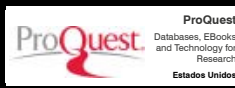

MIคR

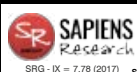

Goosin

Google

ARLA

hi. https://www.mendeley.com/profles/revista-de-arquitectura-bogot/

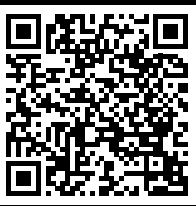

ISSN: 1657-0308 